\title{
Temporal encoding strategies result in boosts to final free recall performance comparable to spatial ones
}

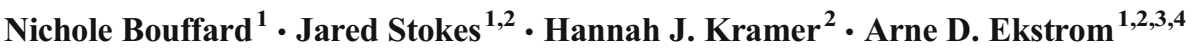

Published online: 25 July 2017

(C) Psychonomic Society, Inc. 2017

\begin{abstract}
The method of loci is a highly effective mnemonic that recruits existing salient memory for spatial locations and uses the information as a scaffold for remembering a list of items (Yates, 1966). One possible account for the effectiveness of the spatial method of loci comes from the perspective that it utilizes evolutionarily preserved mechanisms for spatial navigation within the hippocampus (Maguire et al. in Proceedings of the National Academy of Sciences, 97(8), 4398-4403, 2000; O'Keefe \& Nadel, 1978; Rodriguez et al. in Brain Research Bulletin, 57(3), 499-503, 2002). Recently, though, neurons representing temporal information have also been described within the hippocampus (Eichenbaum in Nature Reviews Neuroscience, 15(11), 732-744, 2014; Itskov, Curto, Pastalkova, \& Buzsáki in The Journal of Neuroscience, 31(8), 2828-2834, 2011; MacDonald, Lepage, Eden, \& Eichenbaum in Neuron, 71(4), 737-749, 2011; Mankin et al. in Proceedings of the National Academy of Sciences, 109(47), 19462-19467, 2012; Meck, Church, \& Matell in Behavioral Neuroscience, 127(5), 642, 2013), challenging the primacy of spatial-based functions to hippocampal processing. Given the presence of both spatial and temporal coding mechanisms within the hippocampus, we predicted
\end{abstract}

Arne D. Ekstrom

adekstrom@ucdavis.edu

1 Center for Neuroscience, University of California, Davis, 1 Shields Ave, Davis, CA 95618, USA

2 Department of Psychology, University of California, Davis, 1 Shields Ave, Davis, CA 95618, USA

3 Neuroscience Graduate Group, University of California, Davis, 1 Shields Ave, Davis, CA 95618, USA

4 Department of Psychology and Center for Neuroscience, University of California, Davis, 1544 Newton Ct., Davis, CA 95618, USA that primarily temporal encoding strategies might also enhance memory. In two different experiments, we asked participants to learn lists of unrelated nouns using the (spatial) method of loci (i.e., the layout of their home as the organizing feature) or using two novel temporal methods (i.e., autobiographical memories or using the steps to making a sandwich). Participants' final free recall performance showed comparable boosts to the method of loci for both temporal encoding strategies, with all three scaffolding approaches demonstrating performance well above uninstructed free recall. Our findings suggest that primarily temporal representations can be used effectively to boost memory performance, comparable to spatial methods, with some caveats related to the relative ease with which participants appear to master the spatial versus temporal methods.

Keywords Method of loci $\cdot$ Spatial navigation · Temporal · Sequence learning $\cdot$ Episodic memory

Everyone has experienced learning something (e.g., a phone number) and then, almost immediately, finding that information lost forever, incapable of being retrieved. To combat these lapses in memory and to facilitate learning, humans have developed metacognitive strategies to aid in both the encoding and recall of information. One particularly effective strategy, the method of loci, involves retrieving salient spatial memories, usually a highly familiar spatial environment (like locations around your house), and using the spatial loci, or landmarks, within the representation as slots or scaffolds for individual list items (Yates, 1966). Thus, recall for a list of words is facilitated by mentally traveling a route through the environment and revisiting the locations, thereby cueing the previously placed list item, suggesting that spatial-based strategies are optimal for encoding novel items. 
Research by Roediger (1980) compared multiple different list learning strategies and found that when participants were given sufficient training, the method of loci, along with the peg method, were more effective than other strategies such as rehearsal, imagery, and the link method (mentally linking an image of each item with the images of neighboring list items), specifically for recalling a list in the same order as it was presented. A peg system, or peg method, is a mnemonic in which one memorizes a sequence of images and then uses that learned sequence as pegs to pair with the list items. It is important to note that although both the method of loci and the peg method were comparable in their effectiveness, participants had to study and memorize "the pegs" the day before the experiment. This extra day of memorizing the list of pegs is not needed when using the method of loci, making it a more efficient method to learn and employ. Indeed, even without extensive training, the method of loci appears to be a highly effective strategy for encoding new information. Legge, Madan, Ng, and Caplan (2012) trained participants on the method by reading them a brief description (adapted from Yates, 1966) and then had the participants use the method of loci using either a well-known environment or a novel virtual environment for which they were given 5 minutes to study. With just the brief description, participants were able to effectively implement the mnemonic and memorize multiple lists of words, with no significant difference in recall performance between participants who used a well-known or novel environment. While the exact reasons for its effectiveness remain unclear, there is little debate that it is one of the best mnemonics for encoding and retrieving new memories (Bower, 1970; Bower \& Reitman, 1972; Cornoldi \& De Beni, 1991; Legge et al., 2012; Maguire, Valentine, Wilding, \& Kapur, 2003; Roediger, 1980; Ross \& Lawrence, 1968).

An important aim of cognitive neuroscience is to understand what brain regions are involved in learning and memory. The hippocampus is a brain region that is of particular interest when studying these processes and has been extensively researched in the context of learning and memory. One of the critical roles of the hippocampus is its importance in representing spatial information. Support for this idea comes from the discovery of place cells, which are hippocampal neurons that code or fire for specific locations in space, within the brains of rats (Hollup, Molden, Donnett, Moser, \& Moser, 2001; O'Keefe \& Dostrovsky, 1971; O'Keefe \& Nadel, 1978) and humans (Ekstrom et al., 2003), as well as the finding that human hippocampal lesions impair spatial memory (Astur, Taylor, Mamelak, Philpott, \& Sutherland, 2002; Bartsch et al., 2010; Kolarik et al., 2016). Even though research into the exact mechanisms is ongoing, work from both human and nonhuman fields suggests that the hippocampus is fundamental to spatial navigation and spatial memory. Therefore, the hippocampus is likely one of several brain regions engaged when using the method of loci (Ekstrom,
Arnold, \& Iaria, 2014; Maguire et al., 2003; Maguire et al., 2000).

Another important function of the hippocampus, however, is the encoding and retrieval of autobiographical memories. Autobiographical memories are unique memories for events that occur throughout an individual's life. These detailed memories are composed of many pieces of information; such as when and where the event took place, who the individual was with, what they were doing, and how they were feeling (Brewer, 1989; Conway \& Pleydell-Pearce, 2000). All of these features combine to create a detailed memory that can be relived or reexperienced during recall (Rubin \& Thompson, 1998). Brewer (1989) argued that a key feature of autobiographical memories is the strong self-reference component, which makes autobiographical memories important for creating an individual's identity and the sense of a stable self throughout time (Conway \& Haque, 1999; Conway \& Tacchi, 1996). The self-reference feature also makes these memories distinct from other types of memories such as semantic memory (factual knowledge; Tulving, 1972), for which there is no direct relationship with the self. Evidence from neuroimaging studies suggests that the hippocampus shows greater activation for the recall of autobiographic memories than for the recall of semantic information (Greenberg et al., 2005). Furthermore, patients with damage to the hippocampus also show deficits in autobiographical memory but not in describing aspects of their personality more generally (Rosenbaum et al., 2008). These findings illustrate that the vividness and salience of autobiographical memories likely derives, in part, from strong neural representation of these memories in the hippocampus.

A prominent feature of autobiographical memories is their anchoring in time (Rosenbaum et al., 2008; Tulving, 2002). Within each memory is information about when the event took place in the past, how long ago it took place relative to the present, and how long the event lasted. It is likely that these temporal features are represented in the hippocampus (Addis, Moscovitch, Crawley, \& McAndrews, 2004; Fink et al., 1996; Mankin et al., 2012). There are many levels of temporal processing: milliseconds, hours, days, and even years. Humans therefore have neural mechanisms that allow for a range of finely tuned temporal perception. This ability is critical not only for perceiving the passage of time but for many other processes, such as speech processing, motor movements, and sound localization (Mauk \& Buonomano, 2004). Within the hippocampus, "time cells," or temporal coding neurons, alter their firing rate and spike pattern to code both external temporal information as well as an internal representation of time (Itskov et al., 2011; MacDonald, Lepage, Eden, \& Eichenbaum, 2011; Eichenbaum, 2014) and to code aspects of temporal sequences (Allen, Salz, McKenzie, \& Fortin, 2016). Further, the human hippocampus has also shown changes in activation to temporal intervals (Mankin et al., 2012; Meck et al., 2013) and sequences 
(Ekstrom, Copara, Isham, Wang, \& Yonelinas, 2011; Lehn et al., 2009). This suggests that although the hippocampus is central for spatial processing, it may also play a significant role in representing temporal features, which are an important component of autobiographical memories.

Given that the hippocampus contains neural populations representing both spatial and temporal information, and that the method of loci relies on a spatial encoding strategy, it may be possible to utilize an encoding strategy depending on temporal features, at least partially, in the same way. To our knowledge, however, no previous studies have explicitly investigated the efficacy of using primarily temporal features in an associative mnemonic like the method of loci. Thus, our study aimed to investigate whether a primarily temporal representation could be used effectively as a scaffold in the method of loci by tapping into temporal coding mechanisms, likely supported, in part, by the hippocampus. To test this idea, in Experiment 1, we designed a novel temporal method in which participants created a timeline of autobiographical life memories, as opposed to a frequently traveled route, to act as the scaffold in the mnemonic. We also considered that other representations, such as a well-rehearsed sequence of events, might access a strong temporal structure, which we then investigated in Experiment 2. We note that while autobiographical (Experiment 1) and sequence coding (Experiment 2) emphasize temporal information during encoding, they likely contain some spatial information as well (e.g., when remembering an event on an autobiographic time line, one also likely accesses some information about location (Arzy, Adi-Japha, \& Blanke, 2009). Compared to the spatial method of loci, which emphasizes a spatial scaffold as the primary organizing feature during encoding, the two temporal methods put a greater emphasis on use of a temporal coding strategy. We thus hypothesized that the temporal feature of participants' autobiographical memories would serve as a strong and salient scaffold in the mnemonic and enhance participant performance just as effectively as the spatial method of loci. As a control comparison, we compared the scaffolding strategies with spontaneous, uninstructed free recall.

\section{Experiment 1}

\section{Method}

Participants Thirty-one undergraduate psychology students (seven males) ages 18 to 26 years $(M=20.92, S D=1.67)$ from the University of California, Davis, participated in this study. Our sample size of 31 approximates those used in other studies (e.g., Roediger, 1980) that observed significant effects of the method of loci on learning (note that because we employed a within-, rather than between-subjects design, we only required a number of participants comparable to a single condition in Roediger, 1980, and expected greater statistical power overall due to a within-subjects design). Because no previous studies have compared the two methods in a within-subjects design and the temporal method is completely novel, all sample size was based on estimates from past work. All participants were compensated with course credit for participating. The study consisted of a single visit and took approximately 1 hour to complete. All participants were right handed and native English speakers. One participant was excluded for failure to complete the experiment.

Procedure Participants were seated in front of a computer and told they would be memorizing lists of words. The lists were created using nouns from the Toronto Noun Pool. ${ }^{1}$ Each list consisted of 10 unrelated nouns (i.e., "TOOL," "DOLL," "FLAME," "HAT," etc.). First, every participant completed the uninstructed ("free") task in which they were told to simply remember the words. After memorizing two lists in the free task, participants were told they were going to be trained on an effective memory technique that they were to use on the subsequent lists. Every participant was trained on both autobiographic temporal and spatial methods. We counterbalanced the order that participants learned and used each method (spatial first: $N=15$, temporal first: $N=15$ ) so that any behavioral differences observed were not simply due to always experiencing one of the methods first or last. After correctly memorizing and recalling the two lists using the first method, participants were then trained on the second method. Participants who had first been trained on the spatial method of loci were trained on the autobiographic temporal method and vice versa. They learned two new lists using the second method (see Fig. 1 for a schematic). Last, participants completed a final free recall task, in which they wrote down as many words as they could remember from all six lists.

Free (uninstructed) task. Participants completed the free task first. They were shown a list of 10 words, one at a time, and were not instructed to use any particular strategy when memorizing the list. Each word appeared on the screen for 3 seconds, with an interstimulus interval of 3 seconds. After the 10th word, participants responded to the question, "How difficult was this task?" using a Likert-type scale $(1=$ not at all difficult, $4=$ very difficult). This question was followed by a distractor task: five simple math equations (addition, subtraction, or multiplication), which appeared on the screen with either a correct or incorrect solution, and participants evaluated

\footnotetext{
${ }^{1}$ http://memory.psych.upenn.edu/files/wordpools/nouns.txt. Accessed on December 15, 2014.

It is important to note that all of nouns were concrete nouns, with high imagery scores. The method of loci is most efficient for improving recall of concrete nouns compared to abstract nouns, or words incapable of being visualized (Legge et al., 2012).
} 


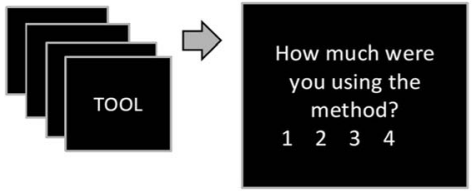

Fig. 1 This schematic represents the paradigm for a list in the spatial, autobiographic temporal (Experiment 1), and procedural temporal (Experiment 2) tasks. Each word appeared on the screen for a total of 3 seconds, followed by a 3 second interstimulus interval. Participants responded to the question "How much were you using the method?" using a Likert-type scale. Participants then responded to five math

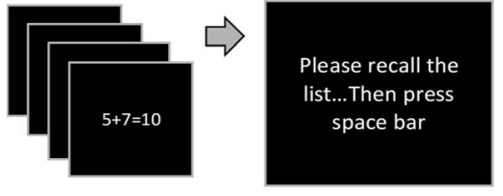

Repeat until all 10 words are recalled

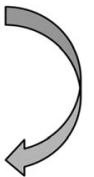

problems (pressing " 1 " for correct and " 2 " for incorrect). Last, they were prompted on the screen to verbally recall the entire list of words. Participants repeated this procedure until all 10 words were recalled. Note. The design was the same for the free task, except the probe question was different

layout/route was taken away. The experimenter read a practice list of five words to the participant, and after each word they verbalized how they were going to place each word along their route to ensure that the participant was using the method correctly. During this time, they were given feedback and were corrected if necessary. They proceeded to use the spatial method to memorize two new lists of words.

The paradigm for the spatial method of loci task was otherwise identical to the free (uninstructed) task, except that the question that appeared on the screen after the 10th word, which was changed to "How much were you using the method?", and responses were again made using a Likert-type scale $(1=$ not at all, $4=$ the whole time $)$.

Autobiographic temporal method task. The autobiographic temporal method task is the novel method designed to emphasize a temporal feature as the scaffold. For this method, participants were handed a piece of paper and instructed to create a chronological timeline using 10 of their biggest, most memorable and detailed memories. During training, participants were instructed to take the words as they appeared on the screen and somehow incorporate them into their memory. Participants were given the following example: "If your memory is 'first time on a train,' and the word on the screen is 'BANANA,' you would then recall your memory from childhood about riding on a train for the first time and imagine sitting on the train and looking out the window, and then add to this by visualizing a giant banana floating by the window." Then participants were instructed to imagine mentally time traveling through their time line of memories during recall and to use that visualization to help them remember and recall the words. The remainder of the task was identical the spatial method of loci task and participants memorized two more lists using the autobiographic temporal method.

Final free recall. At the end of the study, participants took a short break and were asked to describe what methods and strategies they had used during the free task, when they had memorized the first lists before being 
instructed to use a particular strategy. Participants were then handed a piece of paper with six columns, headed "List 1," "List 2," and so on, representing the six lists they had learned throughout the experiment. They wrote down as many words as they could remember from all six lists and placed them in the correct column.

Scoring. To measure participants' performance on both delayed and final free recall lists, we calculated the number of words that participants correctly recalled compared to the original list and plotted the serial position curves (Murdock, 1963). We also plotted conditional response probability (CRPs) as a function of lag in order to measure serial order and forward direction bias in the recalled lists (Kahana, 1996).

For the delayed free recall lists, we calculated the number of words that were correctly recalled and averaged across lists that were learned using the same method. Since all participants repeatedly viewed the lists and performed delayed free recall until they reached criterion, we were most interested in the recall performance during the first recall attempt of each list. Therefore, we conducted all of the following delayed free recall analyses on the first trial of each list. When calculating the number of words recalled during the final free recall lists, one point was given for every correctly recalled word that was placed in the correct list. Points were not given to words that were correctly recalled but placed in the wrong list. We averaged the two recall scores for lists that were learned using the same method. This resulted in a score for each of the methods: free, autobiographic, and spatial.

Given that the spatial method of loci is especially superior in aiding recall in the exact order that items were presented (Roediger, 1980; Yates, 1966), we investigated the serial order of the recalled lists by plotting serial position curves and conditional response probability as a function of lag (or CRP; Kahana, 1996; Murdock, 1963). We calculated serial position curves via the probability of a word being recalled in the correct position. For the CRP as a function of lag, we computed the conditional probability for all possible lag values in the list. For example, given a correctly recalled word, we calculated the probability that the next correctly recalled word would occur at the different possible lag values. In other words, the lag value represents the position of the next word that was recalled by the participant, relative to any successfully recalled word (i.e., lag 0). For example, if a participant successfully recalled the word "DOLL," a lag value of +1 would represent the word that appeared right after "DOLL" in the list (i.e., forward direction), whereas a lag value of -1 would represent the word that came right before "DOLL" in the list (backward direction). Because we were most interested in the difference between the three methods in their ability to enhance recall in serial order (forward direction) and we did not have sufficient trial numbers to include the other directions, the statistical analyses of the following CRP analyses include only lag +1 , which nonetheless included the majority of word transitions. Since the lists were 10 words long, there were 18 possible lags ( -9 to 9 ). We computed the CRP for participant's delayed free recall after the first attempt of each list as well as their final free recall at the end of the experiment. (Note that for the final free recall task, some participants did not recall any words from either the free, autobiographic, or spatial lists and therefore could not be included in this analysis. Thus, the serial order analysis of the final free recall task is conducted on data from 22 participants.)

\section{Results}

We investigated which strategies participants used during the uninstructed task, how many trials it took for participants to reach criterion, how many words were recalled during the first delayed free recall trial of each list, how many words were recalled at the end of the experiment, and the serial order of the recalled lists, which we did by plotting the serial position curves and CRPs in both the delayed and final free recall tasks. Because we did not have strong a priori hypotheses regarding which method would result in better versus worse memory, we employed two-tailed post hoc tests with a Bonferroni correction for multiple comparisons across within-subjects conditions. We also tested all null effects by computing the Bayes factor (Rouder, Speckman, Sun, Morey, \& Iverson, 2009). All error bars in figures represent the Bonferroni corrected, withinsubjects confidence interval (as described by Cousineau, 2005).

Free task strategies As shown in Fig. 2a, participants reported using a number of different strategies during the free task. Techniques such as creating a story, association (associating the words with some specific image, event, or each other), counting with their fingers (paired each word with a finger), imagery (picturing the image of the words), acronym (making a word out of the first letter of each word), and chunking (breaking up the list into smaller lists of three to five words). The "other" category includes strategies that a small number of participants used in tandem with the previous mentioned strategies. These include rhyming, pairing semantically related words, remembering how the words sound all together in a string, creating a sentence, or imaging themselves with the word. Notably, only two participants reported using a spatial strategy. We conducted all analyses with and without these participants, and their data did not change the results; therefore, the following results include these participants.

Learning We conducted a 3 (method: free, spatial, autobiographic) $\times 2$ (method order: spatial first, autobiographic first) repeated-measures ANOVA on the average trials to criterion. This analysis resulted in a main effect of method, $F(1,28)=$ $33.08, p<.001, \eta_{\mathrm{p}}{ }^{2}=.54, d=-0.166$, indicating that learning 


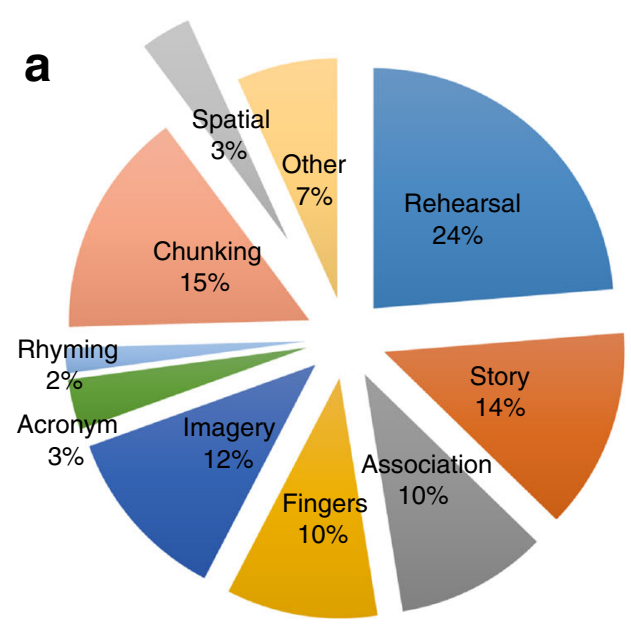

Fig. 2 a The different strategies participants used during the free task in Experiment 1. Percentages are out of the total number of different strategies used (many participants used multiple methods). Majority of participants used techniques that involved some type of rehearsal, or repeating the words over and over again in their head. Notably, only

rate depended on the encoding method employed. There were no significant effects of method order $(F \mathrm{~s}<2.66, p \mathrm{~s}>.09$, $\left.\eta_{\mathrm{p}}{ }^{2} \mathrm{~s}<.09\right)$. Post hoc comparisons revealed that participants took the most trials to learn the list when implementing an uninstructed method ( $M=2.43$ trials to criterion, $p \mathrm{~s}_{\text {corrected }}<$ $.021)$ and learned the list in the least trials when using the spatial method of loci $\left(M=1.38\right.$ trials to criterion, $p \mathrm{~s}_{\text {corrected }}$ $<.001)$. The average trials to criterion for the autobiographic method $(M=2.06)$ fell between the uninstructed method and the spatial method $\left(p \mathrm{~s}_{\text {corrected }}<.001\right)$.

Delayed free recall We conducted a 3 (method: free, spatial, autobiographic) $\times 2$ (method order: spatial first, autobiographic first) repeated-measures ANOVA on the number of words vocally recalled after the first trial of each list. There was a main effect of method, $F(1,28)=19.50, p<.001, \eta_{\mathrm{p}}{ }^{2}=.41$, qualified by a Method $\times$ Method Order interaction, $F(1,28)=$ $4.24, p=.02, \eta_{\mathrm{p}}{ }^{2}=.13$. Participants recalled significantly more words when they were using the spatial method $(M=$ $\left.9.36, p \mathrm{~s}_{\text {corrected }}<.001\right)$ than when they were using uninstructed methods $(M=7.57)$ or the autobiographic method $(M=7.61)$. Participant's performance on the autobiographic lists was more similar to the uninstructed lists $(t=-.104)$. The Bayes factor calculated for the delayed free recall for autobiographic versus uninstructed methods suggests (with moderate evidence) that these results were most likely obtained under the null hypothesis that there was no difference in number of words recalled $\left(\mathrm{BF}_{10}=.195\right)$. These findings suggested that participants were quick to adopt the spatial method of loci and use it effectively to recall words after the first trial of each list in comparison to the autobiographic (or free) method.

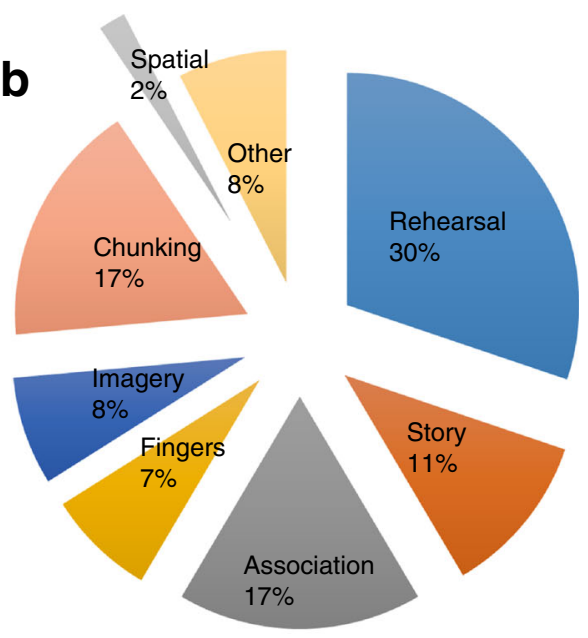

two participants reported using a spatial strategy (depicted as 3\% of the total strategies in the figure). b Free recall strategies reported from Experiment 2. Only one participant reported using a spatial strategy (depicted as $2 \%$ of the total strategies). (Color figure online)

Final free recall A 3 (method: free, spatial, autobiographic) $\times$ 2 (method order: spatial first, autobiographic first) repeatedmeasures ANOVA examined the average number of words participants correctly recalled at the end of the session during final free recall. This analysis yielded a main effect of encoding method, $F(1,28)=37.09, p<.001, \eta_{\mathrm{p}}{ }^{2}=.57, d=$ .287 , qualified by a Method $\times$ Method Order interaction, $F(1$, 28) $=5.31, p=.02, \eta_{\mathrm{p}}^{2}=.16$. The Method $\times$ Method Order interaction effect appeared to be driven by long-term recency effects, that is, more words were remembered from the lists that were encoded last, regardless of method (Bjork \& Whitten, 1974). We thus did not consider this effect further. The main effect of method is shown in Fig. 3. Across method order, participants recalled more words while using the spatial $(M=8.5)$ and autobiographic $(M=8.01)$ methods than when using an uninstructed method $\left(M=4.33, p s_{\text {corrected }}<.001\right)$. Importantly, post hoc comparison of the number of words recalled using the spatial and autobiographic were not significantly different $(t=.913)$. The Bayes factor calculated for the final free recall for autobiographic versus spatial methods suggests (with moderate evidence) that these results were most likely obtained under the null hypothesis that there was no difference in number of words recalled $\left(\mathrm{BF}_{10}=.284\right)$. The main effect appeared largely driven by better memory for words encoded using either a spatial or autobiographic strategy compared to the "free" procedure. Thus, while our delayed recall tests indicated that the autobiographic was initially inferior to the spatial method, by final recall, participants appeared to have "caught up" and used both effectively.

One possibility, however, that runs counter to the above interpretation is that the effects of encoding strategy on final free recall were driven by repetitions of a list during learning 


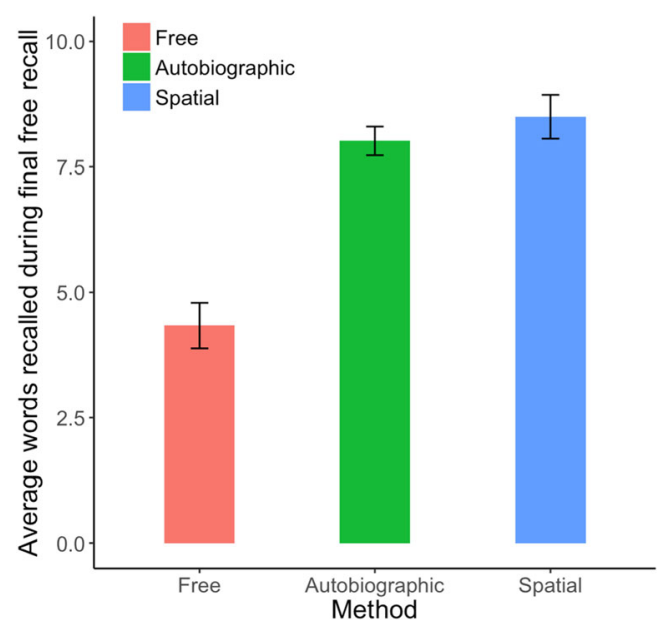

Fig. 3 The average number of words correctly recalled (i.e., placed in the correct list) during the final free recall task in Experiment $1(N=30)$. Scores are out of a total of 10 words. Participants recalled significantly fewer words from the free lists and recalled an equal number of words from both the autobiographic and spatial lists. The error bars are withinsubject Bonferroni-corrected confidence intervals (Cousineau, 2005). (Color figure online)

rather than the organization of the words in memory. To test this possibility, we examined correlations between the number of trials participants took to reach criterion and their subsequent average recall for those lists. After correcting for multiple comparisons, none of these correlations were significant. Therefore, there is no evidence that repeated viewing of the lists during learning enhances long-term recall.

Serial order We plotted the serial position curves for participants' delayed and final free recall to determine the accuracy of their order memory. As shown in Fig. 4a, the serial position curve for the first recall trial illustrates a higher probability of recall of words in the correct position for the spatial lists. It is also notable that the autobiographic lists seem to have a boost in recall compared to free lists, especially for words near the end of the list. We also plotted the serial position curve for the final free recall lists (see Fig. 4b). Here we found that autobiographic and spatial lists showed a greater probability of recall than the free lists.

To answer more complex questions about the serial order memory, we also computed CRP as a function of lag for participants' delayed and final free recall. A 3 (method: free, spatial, autobiographic) $\times 1(\operatorname{lag}+1)$ repeated-measures ANOVA on the CRP for participants first delayed free recall trial yielded a main effect of method, $F(1.84,53.24)=22.63, p<.001, \eta_{\mathrm{p}}{ }^{2}=.44$. As shown in Fig. 4c, participants showed a greater bias for recalling words in the correct serial order when they were using the spatial method compared to when they were using either the uninstructed or autobiographic method $\left(p \mathrm{~s}_{\text {corrected }}<\right.$ .001). There was no significant difference in CRP at lag
+1 for the uninstructed and autobiographic method ( $p$ corrected $=.238)$. The Bayes factor calculated for the CRP at lag +1 for autobiographic versus spatial methods during the first recall attempt suggests (with anecdotal evidence) that these results were most likely obtained under the null hypothesis that there was no difference in CRP $\left(\mathrm{BF}_{10}=\right.$ .792). Thus, these findings suggest that initially, just like the number of words recalled, the spatial method showed the highest boost in order memory.

We conducted the same analysis on the conditional response probability for participants final free recall. This analysis yielded a main effect of method, $F(1.74$, $36.54)=11.56, p<.001, \eta_{\mathrm{p}}^{2}=.24$. As shown in Fig. $4 d$, participants showed a similar bias for recalling words in the correct serial order when they were using the both autobiographic and spatial methods $\left(p_{\text {corrected }}=\right.$ .675). The Bayes factor calculated for the CRP at lag +1 for autobiographic versus spatial methods during final free recall suggests (with anecdotal evidence) that these results were most likely obtained under the null hypothesis that there was no difference in CRP $\left(\mathrm{BF}_{10}=\right.$ .434). Both autobiographic and spatial methods showed greater CRP for lag +1 than the uninstructed method $\left(p \mathrm{~s}_{\text {corrected }}<.004\right)$. Together, these findings suggest that forward direction bias was stronger for both the autobiographic and spatial lists then for the free uninstructed lists.

\section{Discussion}

Perhaps the most important findings of Experiment 1 is that spatial and autobiographic method produced comparable levels of boosts in both item and serial order memory by the end of the study. Both encoding techniques resulted in significantly better levels of retention compared to uninstructed free recall. Participants' enhanced final free recall performance for lists learned using the autobiographic temporal method suggests that a temporal scaffold can be used effectively to recall list items in the same order as they were presented, which may be due to the strong temporal representation of autobiographical memories in brain regions like the hippocampus. These data suggest that the autobiographic temporal method is a viable means of boosting recall performing on par with the spatial method of loci, at least when considering final free recall performance.

However, there are some important caveats to our finding. First, an analysis of the number of trials to criterion needed to learn each list revealed that although participants took less time to learn lists when using the autobiographic temporal method relative to an uninstructed method, participants reached criterion quicker when using the spatial method than when using the autobiographic temporal method. Second, another important difference between the two methods is that the 

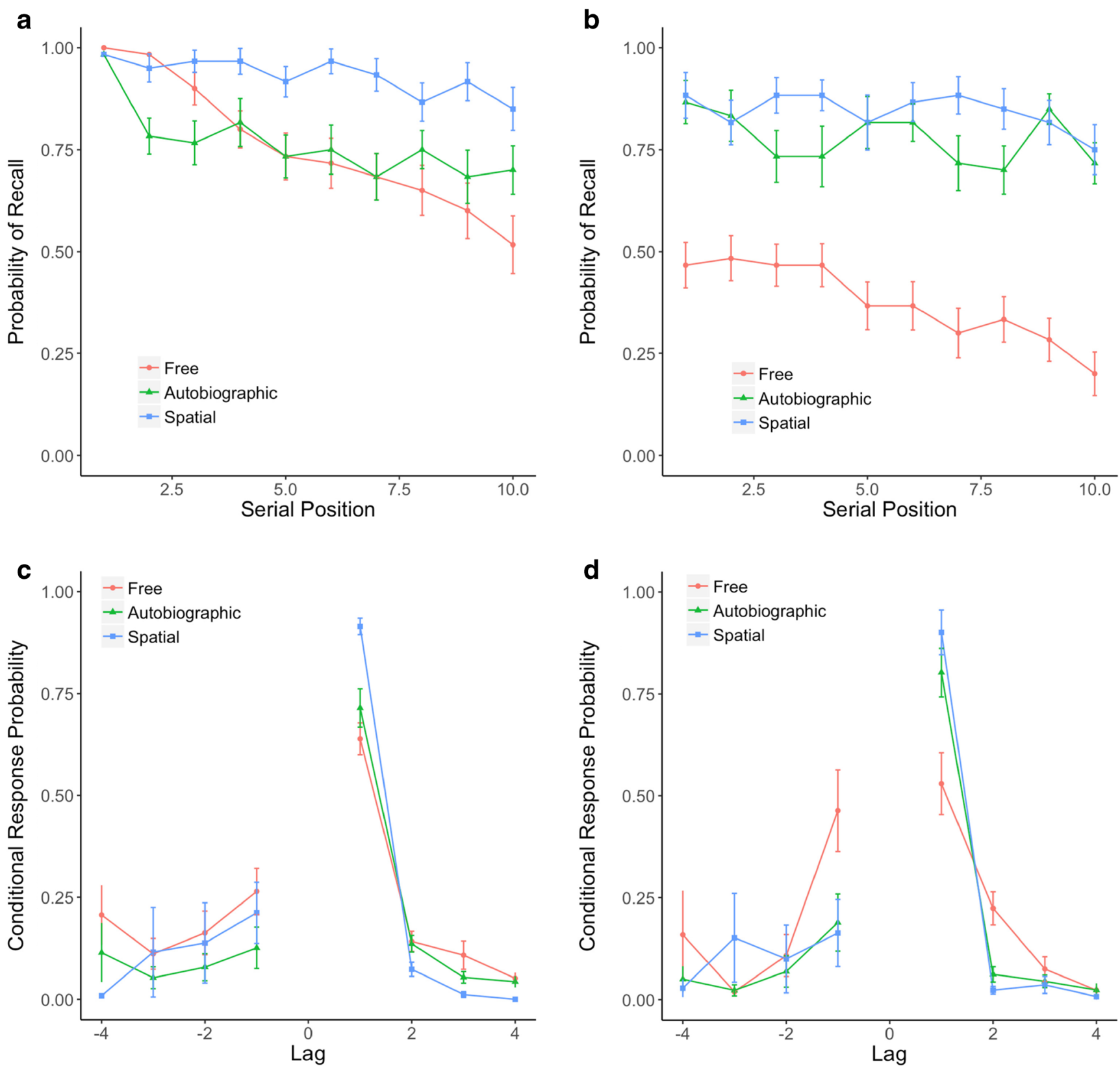

Fig. 4 a The serial position curves for lists recalled during the first delayed free recall trial of in Experiment $1(N=30)$. Participants show a greater probability of recalling words in the correct serial position for spatial lists compared to free and autobiographic lists. b The serial position curves for lists recalled during the final free recall task in Experiment $1(N=30)$. Participants show a greater probability of recalling words in the correct serial position for autobiographic and spatial lists compared to free lists. c The CRP as a function of lag for

autobiographic temporal method did not have the same lag +1 bias that the spatial method did during the first delayed free recall trial of each list. This is a somewhat surprising finding, given that we might expect a temporal encoding strategy to lead to superior temporal order organization, and therefore a stronger lag +1 bias.

the first delayed free recall trial Experiment $1(N=30)$. Participants show a greater CRP at lag +1 for spatial lists compared to free and autobiographic lists. d The CRP as a function of lag for lists recalled during the final free recall task in Experiment $1(N=22)$. Participants show a greater CRP at lag +1 for autobiographic and spatial lists compared to free lists. All error bars are within-subject Bonferronicorrected confidence intervals. (Color figure online)

\section{Experiment 2}

It is possible that the relative difficulty participants had with the autobiographic temporal method compared to the spatial method, was due to a lack of salient temporal features linking memories together. We thus designed a second experiment 
with a different, potentially more accessible temporal feature: the steps to making a sandwich. The steps are events that are fixed in time, well-rehearsed, and familiar to most people. Presumably, each event has a stronger temporal connection to the other steps (i.e., one step cannot come before the next) than autobiographical memories have with each other.

\section{Method}

Participants Thirty-one undergraduate psychology students (nine males) ages 18 to $29(M=20.72, S D=2.50)$ from the University of California, Davis, participated in this study. They were compensated with course credit for participating. The experiment lasted approximately 1 hour. All participants were right handed and native English speakers. Three participants were excluded for not finishing the experiment or failing to follow instructions.

Procedure The paradigm was identical to Experiment 1 with the only difference being the instructions for the temporal method. Twenty-eight participants completed the free task first and then completed the spatial method of loci task (described above in Experiment 1) and the novel procedural temporal method task (procedural first: $N=13$; spatial first: $N=$ 15). All participants ended their session by completing the final free recall task.

Procedural temporal method task. The procedural temporal method was similar to the previous autobiographic temporal method (see Fig. 1), except this time the participant created a sequence of logical steps to make a sandwich. The participants were told to use the method by pairing each word with a step. Participants were given the following example: "If the step is getting two slices of bread out of the bag, and the word is 'BASKET,' imagine taking the slices of bread out of a basket." Participants were instructed to imagine completing each step during recall to help them remember the words. After the training participants used the procedural temporal method to memorize two word lists.

\section{Results}

Free (uninstructed) recall As shown in Fig. 2b, participants used a number of different strategies during the free task, or uninstructed period. Notably, only one participant reported using a spatial strategy. We conducted all analyses with and without this participant and this did not change the results, so we included the participant in subsequent analyses.

Learning We conducted a 3 (method: free, spatial, procedural) $\times 2$ (method order: spatial first, procedural first) repeated- measures ANOVA on the average trials to criterion. This analysis resulted in a main effect of method, $F(1,26)=29.12, p<$ $.001, \eta_{\mathrm{p}}{ }^{2}=.53, d=-.133$. There were no effects of method order $\left(F \mathrm{~s}<3.37, p \mathrm{~s}>.06, \eta_{\mathrm{p}}{ }^{2} \mathrm{~s}<.11\right)$. Participants took the longest to learn the list when implementing an uninstructed method $\left(M=2.78\right.$ trials to criterion, $\left.p \mathrm{~s}_{\text {corrected }}<.001\right)$. Participants learned the lists the fastest when using the spatial method of loci $\left(M=1.46\right.$ trials to criterion, $\left.p \mathrm{~s}_{\text {corrected }}<.003\right)$. The procedural method ( $M=2.05$ trials to criterion) fell between the uninstructed and spatial methods $\left(p \mathrm{~s}_{\text {corrected }}<\right.$ .0028).

Delayed free recall We conducted a 3 (method: free, procedural, spatial) $\times 2$ (method order: spatial first, procedural first) repeated-measures ANOVA on the number of words vocally recalled after the first trial of each list. There was a main effect of method, $F(1,26)=17.17, p<.001, \eta_{\mathrm{p}}{ }^{2}=.40$, qualified by a Method $\times$ Method Order interaction, $F(1,26)=4.37, p=.02$, $\eta_{\mathrm{p}}{ }^{2}=.14$. Participants recalled significantly more words during their first attempt when they were using the spatial method $\left(M=9.20, p \mathrm{~s}_{\text {corrected }}<.001\right)$ than when they were using uninstructed methods $(M=7.05)$ or the procedural method $(M=$ 7.16). The Bayes factor calculated for the delayed free recall for procedural versus uninstructed methods suggests (with moderate evidence) that these results were most likely obtained under the null hypothesis that there was no difference in the number of words recalled $\left(\mathrm{BF}_{10}=.203\right)$. These findings again suggest participants more readily employed the spatial method of loci to learn the list of words in their first attempt and that participants performance on the procedural lists was more similar to the uninstructed lists during the first delayed free recall attempt.

Final free recall A 3 (method: free, spatial, procedural) $\times 2$ (method order: spatial first, procedural first) repeatedmeasures ANOVA explored the number of words participants correctly recalled at the end of the session. This analysis resulted in a main effect of method, $F(1,26)=22.28, p<.001$, $\eta_{\mathrm{p}}{ }^{2}=.46, d=.647$, qualified by a Method $\times$ Method Order interaction, $(1,26)=8.16, p=.001, \eta_{\mathrm{p}}{ }^{2}=.24$. The Method $\times$ Method Order interaction effect again appeared to be driven by long-term recency effects (more words were remembered from the lists that were encoded last than any other list, regardless of method; Bjork \& Whitten, 1974). As illustrated in Fig. 5, across method order, participants recalled more words while using the spatial $(M=7.92)$ and procedural $(M$ $=7.26$ ) methods than when using an uninstructed method ( $M$ $\left.=4.44, \mathrm{~s}_{\mathrm{corrected}}<.001\right)$. Importantly, there was no significant difference between the number of words that participants recalled from spatial and procedural lists $(t=1.45)$. The Bayes factor calculated for the final free recall for procedural versus spatial methods suggests (with anecdotal evidence) that these results were most likely obtained under the null 


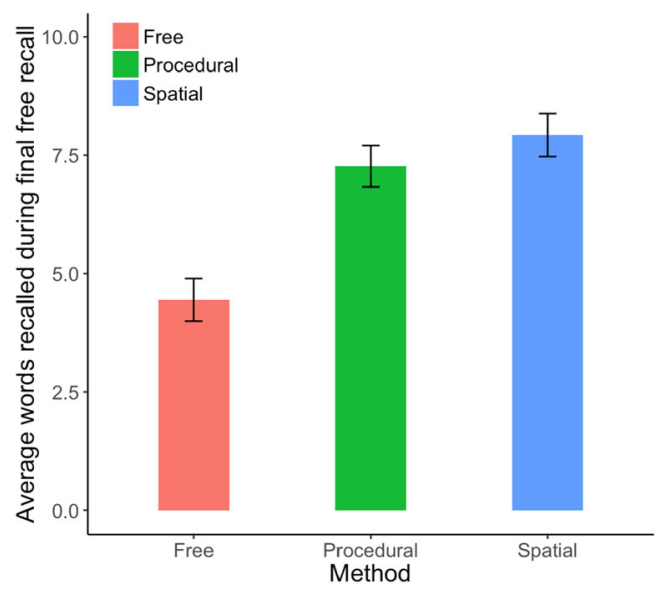

Fig. 5 The average number of words recalled during the final free recall task in Experiment $2(N=28)$. Scores are out of a total of 10 words. Participants recalled significantly fewer words from the free lists and recalled a similar number of words from both the procedural and spatial lists. The error bars are within-subject Bonferroni-corrected confidence intervals. (Color figure online)

hypothesis that there was no difference in number of words recalled $\left(\mathrm{BF}_{10}=.512\right)$.

We examined whether there was a correlation between the average trials to criterion and subsequent average recall for those lists. After correcting for multiple comparisons, no significant correlations remained. This supports our previous hypothesis that more repetitions of a list during learning does not enhance final free recall.

Serial order As shown in Fig. 6a, the serial position curves for the first delayed free recall trial illustrates a higher probability of recall of words in the correct position for the spatial lists. It is also notable that the lists encoded with the procedural method seem to have similar recall probabilities compared to free lists, except for the last two positions in the list. We also plotted the serial position curves for the final free recall lists (see Fig. 6b), and found that, similar to Experiment 1, procedural and spatial lists showed a greater probability of recall than the free lists. A 3 (method: free, spatial, procedural) $\times 1(\mathrm{lag}+1)$ repeatedmeasures ANOVA on the conditional response probability for participants first delayed free recall attempt yielded a main effect of method, $F(1.99,53.77)=13.55, p<.001, \eta_{\mathrm{p}}{ }^{2}=.33$. As shown in Fig. 6c, participants showed a greater bias for recalling words in the correct serial order when they were using the spatial method compared to when they were using either the uninstructed or procedural method $\left(p \mathrm{~s}_{\text {corrected }}<.002\right)$. There was no significant difference in CRP at lag +1 for the uninstructed and procedural method $\left(p_{\text {corrected }}=.588\right)$. The Bayes factor calculated for the CRP at lag +1 for procedural versus spatial methods during the first trial suggested (with anecdotal evidence) that these results were most likely obtained under the null hypothesis that there was no difference in $\mathrm{CRP}\left(\mathrm{BF}_{10}=.432\right)$.
We then conducted the same analysis on the conditional response probability for participant's final free recall, which yielded a main effect of method, $F(1.89,39.79)=17.31, p<$ $.001, \eta_{\mathrm{p}}{ }^{2}=.30$. (Note that some participants did not recall any words from either the free, procedural, or spatial lists and therefore could not be included in this analysis. Thus, the serial order analysis of the final free recall task is conducted on data from 22 participants.) As shown in Fig. 6d, participants showed a similar bias for recalling words in the correct serial order when they were using the both the procedural and spatial methods $\left(p_{\text {corrected }}=.242\right)$. The Bayes factor calculated for the CRP at lag +1 for procedural versus spatial methods during final free recall suggests (with anecdotal evidence) that these results were most likely obtained under the null hypothesis that there was no difference in CRP $\left(\mathrm{BF}_{10}=.866\right)$. Both procedural and spatial methods showed greater CRP for lag +1 than when they were using the uninstructed method ( $p$ $\left.\mathrm{s}_{\text {corrected }}<.001\right)$. These findings suggest that the forward direction bias was stronger for both the procedural and spatial lists then for the free uninstructed lists.

\section{Discussion}

For Experiment 2, we changed how participants employed the temporal method, with the hypothesis that organizing words around the process of making a sandwich would be easier to access and use than the temporal features within a timeline of autobiographical memories. We again found that during final free recall, participants recalled a significantly greater number of words from lists that were learned using the procedural method compared to the uninstructed method and that, similar to the results from Experiment 1, participants showed equivalent recall for lists they had learned using both the procedural temporal and spatial strategies. We again found no significant difference in CRP at lag +1 for procedural temporal and spatial strategies during final free recall. This suggests that the procedural temporal scaffold can be used effectively to enhance recall and also results in a forward direction bias similar to that of the spatial method. We note though that despite our attempts to control for familiarity in the procedural method (i.e., employing a temporal scaffold that participants would be more familiar with, at least on a daily basis), the procedural method still took more trials to master than the spatial method of loci and the CRP at lag +1 during the first delayed free recall trial for procedural lists was not as high as the CRP for spatial lists.

\section{Experiments 3a and 3b}

One limitation to our study is that the first two uninstructed lists were not included in the counterbalance, meaning every participant completed the free (uninstructed) task first and the 

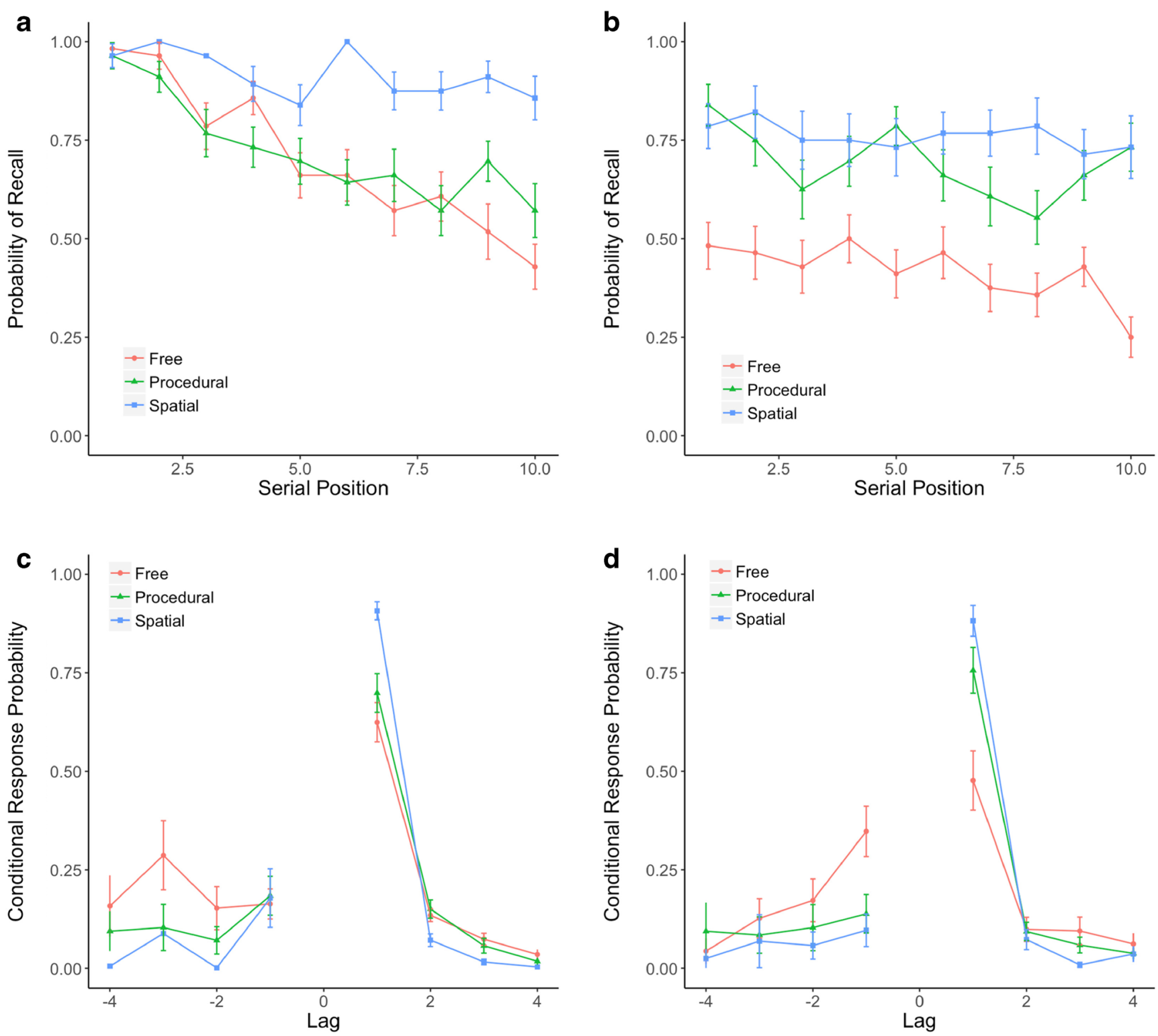

Fig. 6 a The serial position curves for lists recalled during the first delayed free recall trial in Experiment $2(N=28)$. Participants show a greater probability of recalling words in their correct serial position for spatial lists compared to procedural and free lists. b The serial position curves for lists recalled during the final free recall task in Experiment $2(\mathrm{~N}$ $=28$ ). Participants show a greater probability of recalling words in their correct serial position for procedural and spatial lists compared to free lists. $\mathbf{c}$ The CRP conditional response probability as a function of lag for

the first delayed free recall trial in Experiment $2(N=28)$. Participants show a greater CRP at lag +1 for spatial lists compared to procedural and free lists. $\mathbf{d}$ The CRP as a function of lag for the final free recall task in Experiment $2(N=22)$. Participants show a greater CRP at lag +1 for procedural and spatial lists compared to free lists. All error bars are within-subject Bonferroni-corrected confidence intervals. (Color figure online)

other mnemonic tasks later. This was a necessary constraint to ensure that they did not use a spatial or temporal strategy inadvertently or through habit when studying the free list. This means, though, that the "learning-to-learn effect," or the possibility that participants inevitably got better at learning lists as they progressed through the experiment, cannot be eliminated as a possible explanation for our enhanced recall

results. We therefore conducted two control experiments (3a and $3 \mathrm{~b}$ ) where participants learned all six lists using uninstructed methods. Participants completed the list-learning task; however, they were not instructed to use any particular mnemonic when learning and remembering the lists. In Experiment 3a, 13 participants recalled the lists in any order, and in Experiment 3b, 13 participants were specifically 
instructed to recall the lists in serial order (note that one subject was given the wrong final recall sheet and is therefore excluded from the following analyses).

We focus on comparisons with rates of learning because our primary concern was that not fully counterbalancing uninstructed recall would have affected the rate of learning. To test if there was a significant difference in trials to criterion for free and serial list learning, we conducted a 2 (experiment: $3 \mathrm{a}$, 3b) $\times 3$ (list group: first, second, third) repeated-measures ANOVA on the number of trials taken to reach criterion. There was no main effect of experiment, $F(1,23)=.94, p=$ $.34, \eta_{\mathrm{p}}{ }^{2}=.04$, or list group, $F(1,23)=2.77, p=.09, \eta_{\mathrm{p}}{ }^{2}=.11$. These findings suggest that participants did not differ in number of trials to criterion when recalling the lists in any order versus recalling in serial order. Because we did not find any significant difference between the two experiments, we treated the two groups as one control group $(N=25)$. We conducted a 3 (experiment: 1, 2, 3a+b) $\times 3$ (list group: first, second, third) repeated-measures ANOVA on the number of trials to criterion. This analysis yielded a main effect of experiment $F(2,80)$ $=6.76, p<.01, \eta_{\mathrm{p}}{ }^{2}=.14$, and list group, $F(2,80)=32.41, p<$ $.001, \eta_{\mathrm{p}}{ }^{2}=.29$, qualified by an Experiment $\times$ List group interaction, $F(2,80)=2.85, p<.05, \eta_{\mathrm{p}}{ }^{2}=.07$. Participants took a similar number of trials to reach criterion in the first list group, across all three experiments $\left(M_{\exp 1}=2.43, M_{\exp 2}=\right.$ $\left.2.78, M_{\exp 3}=2.82, p \mathrm{~s}_{\text {corrected }}>.275\right)$, but not for the second group of lists $\left(M_{\exp 1}=1.86, M_{\exp 2}=1.80, M_{\exp 3}=2.48, p-\right.$ $\left.\mathrm{s}_{\text {corrected }}<.05\right)$, nor the third $\left(M_{\exp 1}=1.58, M_{\exp 2}=1.71\right.$, $M_{\exp 3}=2.48, p \mathrm{~s}_{\text {corrected }}<.01$ ), which were acquired faster for Experiments 1 and 2. These findings suggest that across all three list groups, participants do not get faster at learning the lists unless they were employing a mnemonic. Thus, these findings support the idea that mnemonics, either the spatial method of loci or temporal methods, significantly improved the rate at which lists were acquired, and that this effect could not be accounted for purely by a learning-to-learn effect alone.

\section{General discussion}

The spatial method of loci is widely regarded as a superior memory method. Given the evidence of temporal coding mechanisms in the hippocampus (Allen et al., 2016; Ekstrom et al., 2011; Itskov et al., 2011; Lehn et al., 2009; Mankin et al., 2012; Mauk \& Buonomano, 2004; Meck et al., 2013), however, we hypothesized that a mnemonic relying on a primarily temporal scaffold could also be used effectively and efficiently to memorize a list of words, an idea that, to our knowledge, has never been reported in the literature previously. We were also interested in investigating how a mnemonic that relies primarily on temporal features compares to one that relies primarily on spatial features, so we designed two novel methods that use temporal scaffolds (autobiographical memories and steps to making a sandwich) and compared participant performance with the novel methods to the traditional spatial method of loci.

Across two studies, our results indicate that both temporal methods resulted in comparable levels of final free recall compared to the spatial method of loci. We found that the autobiographic temporal and the procedural method increased final free recall performance equivalent to the spatial method. Importantly, both temporal methods showed a significant lag +1 bias in CRP, suggesting that they were effective in enhancing recall of the words in serial order (i.e., the same order as the words were presented). It is also important to note that despite our attempts to control for familiarity in the procedural method, we did not find a difference in either item or order memory between the two temporal methods. These findings suggest that temporal methods are capable of enhancing memory for lists of words as effectively as the spatial method of loci. Given that delayed memory retrieval is often used as a hallmark of hippocampal function in many neuropsychological tests and a diagnostic feature of amnesia (Aly, Ranganath, \& Yonelinas, 2013; Squire, Stark, \& Clark, 2004), our findings support the idea that a primarily spatial "scaffold" (often thought to depend on the hippocampus) is not exclusively effective in boosting delayed recall and that other hippocampally based mnemonics are also effective.

Given that we did not perform neuroimaging in this study, we cannot be certain that the hippocampus was recruited for both spatial and temporal methods of encoding. Past work, though, has argued that spatial navigation is an evolutionarily preserved mechanism that relies on the hippocampus (Maguire et al., 2000; O'Keefe \& Nadel, 1978; Rodriguez et al., 2002). There have also been studies suggesting that the hippocampus is a core structure that is activated when participants employ the spatial method of loci (Maguire et al., 2003). Specifically, in their study, Maguire et al. recruited the top competitors from the World Memory Championships, finding that although the memory champions did not show any anatomical or IQ differences, their performance during the memory task did evoke greater activation in the right hippocampus, medial parietal cortex, and retrosplenial cortex compared to control participants. The authors concluded that the greater activation of these brain regions in memory champions, particularly the hippocampus, was due to their expert use of the spatially dependent mnemonic, which resulted in superior memory performance. Given our finding of equivalent final free recall performance with primarily temporal encoding methods and the recent linkages of the hippocampus to temporal coding (Allen et al., 2016; Ekstrom et al., 2011; Itskov et al., 2011; Lehn et al., 2009; MacDonald et al., 2011; Mankin et al., 2012; Mauk \& Buonomano, 2004; Meck et al., 2013), our current findings challenge the perspective that the success of the method of loci is solely due to its putative linking with evolutionarily 
conserved spatial mechanisms in the hippocampus. Instead, our study provides an important advance by suggesting that there are other neural mechanisms that effectively boost memory for both items and serial order, particularly during final free recall.

Given that the spatial method of loci was mastered more quickly by participants during learning than either temporal method, despite our attempts to control for familiarity in Experiment 2, our study supports the idea that this is still the superior approach for encoding novel items. This does not come as a surprise, and instead supports the long-standing belief that the spatial method of loci is one of, if not the best memory mnemonic, overall. We can also conclude, however, that people are capable of using a temporal scaffold in a memory mnemonic like the method of loci to produce overall comparable boosts in final free recall. It is possible that the observed differences in encoding between the spatial and temporal methods highlight a critical difference in how each of these methods draws on the function of the hippocampus, although past work suggests that both spatial and temporal order retrieval recruit the hippocampus to comparable degrees (Ekstrom et al., 2011). It could also be that the two scaffolds we chose access temporal representations that rely on brain regions outside of the hippocampus, so although they have a unique temporal structure, their representation may be weaker or less accessible. A future imaging study is needed to investigate the activation of the hippocampus during both temporal methods (autobiographic and procedural) and to further examine how different temporal features are represented inside and outside of the hippocampus.

One possible limitation we wish to address is the potential influence of bizarre imagery. Across all methods, participants were encouraged to visualize the list items in vivid and creative ways within their scaffold. This could have encouraged the conjuring of bizarre and unusual images. These types of images are known to affect free recall (Einstein \& McDaniel, 1987; McDaniel \& Einstein, 1986) and possibly enhance memory for the more bizarre visualizations. It is possible that one of the scaffolds facilitated the bizarreness effect more than the other, although there is no reason a priori that one method should disproportionately emphasize distinctiveness over another.

Another limitation is the issue of potential spatial coding during the temporal tasks. Previous research suggests that time maybe coded in a spatial fashion (Arzy et al., 2009; Bonato, Zorzi, \& Umilta, 2012; Majid, Gaby, \& Boroditsky, 2013), so it is possible that there was a spatial element involved in both of the novel temporal methods. Specifically, when employing an autobiographical technique, as in Experiment 1, participants almost certainly recalled some spatial location information. This, though, was likely less pronounced during the procedural method (Experiment 2) because recalling the sequences of steps to making sandwich would likely only involve one spatial location (the location of where one most often makes a sandwich). The fact that we obtained similar results for Experiments 1 and 2 argues against the idea that our findings were simply due to using a spatial strategy, just like the spatial method of loci. In addition, both temporal methods clearly put significant demands on temporal order memory, as noted in many studies investigating temporal coding schemes (Anelli, Ciaramelli, Arzy, \& Frassinetti, 2016; Mauk \& Buonomano, 2004), however, it is difficult to ensure that there are no spatial components when participants employ temporal coding schemes. While it is not clear that autobiographical and procedural methods necessitate interval timing, as suggested by time cells, they certainly require memory for temporal order, which is also thought to depend on the hippocampus (e.g., Ergorul \& Eichenbaum, 2006; Eichenbaum, 2014). In addition, temporal intervals would still be useful for encoding temporal information, particularly in the procedural method, when information occurs in a more structured temporal order fashion compared to recalled autobiographical memories (Allen et al., 2016). Thus, we cannot rule out the idea that participants used some spatial strategies when using temporal methods, and that possibly neither method necessitated temporal interval codes. However, it seems reasonable to conclude that spatial, compared to temporal order demands, would be significantly less in both the autobiographical and procedural methods than the spatial method of loci.

Conversely, one could also argue that the spatial method of loci involved some temporal coding, for example, remembering the order of routes around one's apartment. Indeed, we found significant and immediate boosts in serial order memory with the spatial method of loci, which were unanticipated because our instructions did not emphasize learning the words as a sequence. While both temporal methods eventually "caught up" by final recall, the finding of strong order memory with the spatial method of loci suggests it also provides strong scaffolding for this dimension as well. We wish to emphasize, however, that eventually both "spatial" and "temporal" methods were on par in terms of total numbers of words recalled in the correct order by final recall. Thus, while it seems most parsimonious to conclude that the spatial method of loci boosted order memory more immediately, just like it did item memory, we cannot completely rule out the idea that there is also something "special" about space that simply improves learning faster, although not better in the long run.

\section{Conclusion}

We designed two novel methods of loci that employ the use of two different primarily temporal structures. Participants were not only able to successfully use the methods during encoding but they also received a memory boost when performing a final free recall task after a delay. There were costs to using the temporal methods compared to the well-known spatial 
method, particularly during encoding, so further research is needed to determine the ideal temporal structure to use in this type of mnemonic. Importantly, this study opens the door to many future studies of temporal memory and provides a new method for investigating how time is represented in areas of the brain important for memory, like the hippocampus.

Acknowledgments We would like to thank the participants and the University of California, Davis, Center for Neuroscience, and a special thanks to Colin Kyle and Jennifer Lieberman for their contributions to this project. This project was funded by a NINDS grant to ADE (R01NS076856). HJK was supported by the Predoctoral Training Consortium in Affective Science from the NIMH (201302291).

\section{References}

Addis, D. R., Moscovitch, M., Crawley, A. P., \& McAndrews, M. P. (2004). Recollective qualities modulate hippocampal activation during autobiographical memory retrieval. Hippocampus, 14(6), 752762.

Allen, T. A., Salz, D. M., McKenzie, S., \& Fortin, N. J. (2016). Nonspatial sequence coding in CA1 neurons. The Journal of Neuroscience, 36(5), 1547-1563.

Aly, M., Ranganath, C., \& Yonelinas, A. P. (2013). Detecting changes in scenes: The hippocampus is critical for strength-based perception. Neuron, 78(6), 1127-1137. doi:10.1016/j.neuron.2013.04.018

Anelli, F., Ciaramelli, E., Arzy, S., \& Frassinetti, F. (2016). Prisms to travel in time: Investigation of time-space association through prismatic adaptation effect on mental time travel. Cognition, 156, 1-5.

Arzy, S., Adi-Japha, E., \& Blanke, O. (2009). The mental time line: An analogue of the mental number line in the mapping of life events. Consciousness and Cognition, 18(3), 781-785.

Astur, R. S., Taylor, L. B., Mamelak, A. N., Philpott, L., \& Sutherland, R. J. (2002). Humans with hippocampus damage display severe spatial memory impairments in a virtual Morris water task. Behavioural Brain Research, 132(1), 77-84.

Bartsch, T., Schönfeld, R., Müller, F., Alfke, K., Leplow, B., Aldenhoff, J., ... Koch, J. (2010). Focal lesions of human hippocampal CA1 neurons in transient global amnesia impair place memory. Science, 328(5984), 1412-1415.

Bjork, R. A., \& Whitten, W. B. (1974). Recency-sensitive retrieval processes in long-term free recall. Cognitive Psychology, 6(2), 173189.

Bonato, M., Zorzi, M., \& Umilta, C. (2012). When time is space: Evidence from the mental time line. Neuroscience and Biobehavioral Reviews, 36, 2257-2273.

Bower, G. H. (1970). Analysis of a mnemonic device: Modern psychology uncovers the powerful components of an ancient system for improving memory. American Scientist, 58(5), 496-510.

Bower, G. H., \& Reitman, J. S. (1972). Mnemonic elaboration in multilist learning. Journal of Verbal Learning and Verbal Behavior, 11(4), 478-485.

Brewer, W. (1989). What is autobiographical memory? In D. C. Rubin (Ed.), Autobiographical memory (pp. 25-49). New York, NY: Cambridge University Press.

Conway, M. A., \& Haque, S. (1999). Overshadowing the reminiscence bump: Memories of a struggle for independence. Journal of Adult Development, 6(1), 35-44.

Conway, M. A., \& Pleydell-Pearce, C. W. (2000). The construction of autobiographical memories in the self-memory system. Psychological Review, 107(2), 261.
Conway, M. A., \& Tacchi, P. C. (1996). Motivated confabulation. Neurocase, 2(4), 325-339.

Cornoldi, C., \& De Beni, R. (1991). Memory for discourse: Loci mnemonics and the oral presentation effect. Applied Cognitive Psychology, 5(6), 511-518.

Cousineau, D. (2005). Confidence intervals in within-subject designs: A simpler solution to Loftus and Masson's method. Tutorial in Quantitative Methods for Psychology, 1(1), 42-45.

Eichenbaum, H. (2014). Time cells in the hippocampus: A new dimension for mapping memories. Nature Reviews Neuroscience, 15(11), 732-744.

Einstein, G. O., \& McDaniel, M. A. (1987). Distinctiveness and the mnemonic benefits of bizarre imagery. In M. McDaniel \& M. Pressley (Eds.), Imagery and related mnemonic processes (pp. 78102). New York, NY: Springer.

Ekstrom, A. D., Arnold, A. E., \& Iaria, G. (2014). A critical review of the allocentric spatial representation and its neural underpinnings: Toward a network-based perspective. Frontiers in Human Neuroscience, 8, 803.

Ekstrom, A. D., Copara, M. S., Isham, E. A., Wang, W. C., \& Yonelinas, A. P. (2011). Dissociable networks involved in spatial and temporal order source retrieval. NeuroImage, 2011, 18.

Ekstrom, A. D., Kahana, M. J., Caplan, J. B., Fields, T. A., Isham, E. A., Newman, E. L., \& Fried, I. (2003). Cellular networks underlying human spatial navigation. Nature, 425(6954), 184-188.

Ergorul, C., \& Eichenbaum, H. (2006). Essential role of the hippocampal formation in rapid learning of higher-order sequential associations. Journal of Neuroscience, 26(15), 4111-4117.

Fink, G. R., Markowitsch, H. J., Reinkemeier, M., Bruckbauer, T., Kessler, J., \& Heiss, W. D. (1996). Cerebral representation of one's own past: Neural networks involved in autobiographical memory. Journal of Neuroscience: The Official Journal of the Society for Neuroscience, 16(13), 4275-4282.

Greenberg, D. L., Rice, H. J., Cooper, J. J., Cabeza, R., Rubin, D. C., \& LaBar, K. S. (2005). Co-activation of the amygdala, hippocampus and inferior frontal gyrus during autobiographical memory retrieval. Neuropsychologia, 43(5), 659-674.

Hollup, S. A., Molden, S., Donnett, J. G., Moser, M. B., \& Moser, E. I. (2001). Place fields of rat hippocampal pyramidal cells and spatial learning in the watermaze. European Journal of Neuroscience, 13(6), 1197-1208.

Itskov, V., Curto, C., Pastalkova, E., \& Buzsáki, G. (2011). Cell assembly sequences arising from spike threshold adaptation keep track of time in the hippocampus. The Journal of Neuroscience, 31(8), 28282834.

Kahana, M. J. (1996). Associative retrieval processes in free recall. Memory \& Cognition, 24(1), 103-109.

Kolarik, B. S., Shahlaie, K., Hassan, A., Borders, A. A., Kaufman, K. C., Gurkoff, G., ... Ekstrom, A. D. (2016). Impairments in precision, rather than spatial strategy, characterize performance on the virtual Morris Water Maze: A case study. Neuropsychologia, 80, 90-101.

Legge, E. L., Madan, C. R., Ng, E. T., \& Caplan, J. B. (2012). Building a memory palace in minutes: Equivalent memory performance using virtual versus conventional environments with the Method of Loci. Acta Psychologica, 141(3), 380-390.

Lehn, H., Steffenach, H.-A., van Strien, N. M., Veltman, D. J., Witter, M. P., \& Håberg, A. K. (2009). A specific role of the human hippocampus in recall of temporal sequences. The Journal of Neuroscience, 29(11), 3475-3484.

MacDonald, C. J., Lepage, K. Q., Eden, U. T., \& Eichenbaum, H. (2011). Hippocampal "time cells" bridge the gap in memory for discontiguous events. Neuron, 71(4), 737-749.

Maguire, E. A., Gadian, D. G., Johnsrude, I. S., Good, C. D., Ashburner, J., Frackowiak, R. S., \& Frith, C. D. (2000). Navigation-related structural change in the hippocampi of taxi drivers. Proceedings of the National Academy of Sciences, 97(8), 4398-4403. 
Maguire, E. A., Valentine, E. R., Wilding, J. M., \& Kapur, N. (2003). Routes to remembering: The brains behind superior memory. Nature Neuroscience, 6(1), 90-95.

Majid, A., Gaby, A., \& Boroditsky, L. (2013). Time in terms of space. Frontiers in Psychology, 4, 554.

Mankin, E. A., Sparks, F. T., Slayyeh, B., Sutherland, R. J., Leutgeb, S., \& Leutgeb, J. K. (2012). Neuronal code for extended time in the hippocampus. Proceedings of the National Academy of Sciences, 109(47), 19462-19467.

Mauk, M. D., \& Buonomano, D. V. (2004). The neural basis of temporal processing. Annual Review of Neuroscience, 27, 307-340.

McDaniel, M. A., \& Einstein, G. O. (1986). Bizarre imagery as an effective memory aid: The importance of distinctiveness. Journal of Experimental Psychology: Learning, Memory, and Cognition, 12(1), 54

Meck, W. H., Church, R. M., \& Matell, M. S. (2013). Hippocampus, time, and memory-A retrospective analysis. Behavioral Neuroscience, 127(5), 642.

Murdock, B. B. (1963). The serial position effect in free recall. Journal of Experimental Psychology, 64, 482-488.

Murdock, B. B. (1974). Human memory: Theory and data. Mahwah, NJ: Erlbaum.

O'Keefe, J., \& Dostrovsky, J. (1971). The hippocampus as a spatial map: Preliminary evidence from unit activity in the freely-moving rat. Brain Research, 34(1), 171-175.

O'Keefe, J., \& Nadel, L. (1978). The hippocampus as a cognitive map. New York, NY: Oxford University Press.

Rodriguez, F., López, J. C., Vargas, J. P., Broglio, C., Gómez, Y., \& Salas, C. (2002). Spatial memory and hippocampal pallium through vertebrate evolution: Insights from reptiles and teleost fish. Brain Research Bulletin, 57(3), 499-503.

Roediger, H. L. (1980). The effectiveness of four mnemonics in ordering recall. Journal of Experimental Psychology: Human Learning and Memory, 6(5), 558.

Rosenbaum, R. S., Moscovitch, M., Foster, J. K., Schnyer, D. M., Gao, F., Kovacevic, N., ... Levine, B. (2008). Patterns of autobiographical memory loss in medial-temporal lobe amnesic patients. Journal of Cognitive Neuroscience, 20(8), 1490-1506. doi:10.1162/jocn.2008. 20105

Ross, J., \& Lawrence, K. A. (1968). Some observations on memory artifice. Psychonomic Science, 13(2), 107-108.

Rouder, J. N., Speckman, P. L., Sun, D., Morey, R. D., \& Iverson, G. (2009). Bayesian $t$ tests for accepting and rejecting the null hypothesis. Psychonomic Bulletin \& Review, 16(2), 225-237.

Rubin, D. C., \& Thompson, C. P. (1998). Beginnings of a theory of autobiographical remembering. In C. P. Thompson, D. J. Herrmann, D. Bruce, J. D. Read, D. G. Payne, \& M. P. Toglia (Eds.), Autobiographical memory: Theoretical and applied perspectives (pp. 47-67). Mawah, NJ: Erlbaum.

Squire, L. R., Stark, C. E., \& Clark, R. E. (2004). The medial temporal lobe. Annual Review of Neuroscience, 27, 279-306.

Tulving, E. (1972). Episodic and semantic memory: 1. In E. Tulving \& W. Donaldson (Eds.), Organization of memory (pp. 381-403). New York, NY: Academic Press.

Tulving, E. (2002). Episodic memory: From mind to brain. Annual Review of Psychology, 53, 1-25.

Yates, F. A. (1966). The art of memory. Chicago, IL: The University of Chicago Press. 